\title{
Dificultades en el diagnóstico histológico y en el tratamiento de la enfermedad de Hirschprung
}

\section{Difficulties in Histological diagnosis and treatment of Hirschprung's disease}

\author{
Mario Marcelino More-Flores', \\ Emiliano Paico-Vílchez ${ }^{2}$
}

More-Flores M,Paico-Vílchez E. Dificultades en el diagnóstico histológico y en el tratamiento de la enfermedad de Hirschprung. Rev Soc Peru Med Interna. 2020;33(4): I46-I50.

https://doi.org/10.36393/spmi.v33i4.561

\begin{abstract}
RESUMEN
Tradicionalmente la enfermedad de Hirschsprung, se diagnostica y trata bajo el paradigma de la ausencia de plexos nerviosos submucoso y mientérico en una zona localizada del colon.

El tratamiento quirúrgico consiste en la resección del colon agangliónico y la anastomosis del segmento gangliónico descendido con el ano. La revisión de los resultados a largo plazo de la cirugía, con las diversas técnicas quirúrgicas hasta hoy existentes, muestran complicaciones relacionadas a incontinencia y estreñimiento en cerca del $50 \%$ de los casos.

Muchos cirujanos piensan que esta enfermedad es aún desconocida e incluso incurable.

En base a investigaciones de biología molecular, ahora se conoce que, además de la ausencia de los plexos nerviosos y de las células de Cajal en el segmento agangliónico, existe falla en la secreción de mediadores denominados purinérgicos, como el ATP y sus receptores, así como también de las sustancias nitraérgicas (óxido nítrico), que intervienen en la relajación del músculo intestinal.

Por otro lado, la investigación en el campo de la genética nos ha permitido conocer el rol de las mutaciones de prooncoenes, como el RET y otros, en la génesis de la enfermedad de Hirschsprung.

En el presente trabajo se hace una revisión de los conceptos arriba mencionados y se discuten las dificultades en el diagnóstico histológico de la enfermedad y se propone un enfoque racional e inteligente, encontrado en la literatura revisada.
\end{abstract}

Palabras clave: Enfermedad de Hirschsprung, colon, aganglionar, plexo mientérico.

\section{ABSTRACT}

Traditionally Hirschsprung's disease has been diagnosed and treated under the paradigm of the absence of submucosal and myenteric nerve plexus in a localized area of the colon.

Surgical treatment consists of resection of the aganglionic area and anastomosis of the anal level of the ganglionic region of

I Cirujano pediátrico. Departamento de Biotecnología del Instituto Nacional de Salud del Niño. Profesor de la Universidad Ricardo Palma, Lima, Perú.

2 Cirujano pediátrico. Servicio de Cirugía Pediátrica y del Servicio de Especialidades Quirúrgicas del Hospital Belén de Trujillo. Profesor de la Universidad Privada Antenor Orrego, Trujillo, Perú. the colon. The review of the long-term results of the surgery, with the various surgical techniques existing up to now show complications related to incontinence and constipation in about $50 \%$ of the cases. Many surgeons think that this disease is still unknown and even incurable.

With the advancement in the field of research in molecular biology in the been discovered that addition to the absence of the nerve plexuses, and the Cajal cells in the aganglionic segment, failure in the secretion of mediators called purinergic, such as the ATP and its receptors, as well nitrate substance 
such as nitric oxide, which intervene in the relaxations of the intestinal muscle.

On the other hand, research in the field of genetics has allowed us to know the role of pro-oncogenes mutations such as RET and others, in the genesis of Hirschsprung's disease.

In this paper.A review of the above-mentioned concepts is made and the difficulties in the histological diagnosis of the disease are discussed and a rational and intelligent approach is proposed, found in the reviewed literature.

Keywords: Hirschsprung's disease, colon, aganglionar, myenteric plexus.

\section{INTRODUCCIÓN}

Desde que Harold Hirschsprung describió la enfermedad que lleva su apellido, ella es diagnosticada y tratada bajo el paradigma de la ausencia de los plexos nerviosos submucoso y mientérico en un segmento del colon ${ }^{1,2,3}$, por lo cual se han ideado una serie de técnicas quirúrgicas que consisten en la resección del segmento de colon afectado. Sin embargo, los resultados a largo plazo con las diversas técnicas quirúrgicas existentes son preocupantes pues muestran complicaciones relacionadas a incontinencia y estreñimiento en cerca del $50 \%$ de casos. ${ }^{4,5}$

El presente estudio tiene como finalidad describir las dificultades que hasta hoy presenta el diagnóstico histológico de la enfermedad, la preocupación de diversos cirujanos respecto a sus resultados, los nuevos conocimientos en lo que respecta a la inervación de intestino, el rol de algunas mutaciones genéticas y ciertos criterios que se deben tener en cuenta durante el diagnóstico y tratamiento de la EH.

\section{DIAGNÓSTICO HISTOLÓGICO}

Tradicionalmente se ha considerado que la EH se debe a la ausencia de las células ganglionares en los plexos nerviosos de Auerbach y de Meissner en la pared intestinal. ${ }^{1} \mathrm{La}$ EH afecta habitualmente al recto sigmoides (75\% de los casos); puede presentarse en un segmento largo (15\%); de 1 a $5 \%$ afecta a la región anal (segmento ultracorto); $y$, en el 7,5 \% de los casos afecta a todo el colon y puede extenderse hacia el intestino delgado largo., ${ }^{1,2,3}$

En las biopsias, la demostración de la ausencia de los plexos de Auerbach y Meissner, teñidas con hematoxilina eosina es considerada como el "gold standard" en el diagnóstico de la $\mathrm{EH}$, con una sensibilidad y especificidad casi del 100\%, dependiendo de la calidad de las biopsias, número de secciones examinadas y la experiencia del anatomopatólogo. ${ }^{2}$

Maier y $\mathrm{Rugh}^{5}$ introdujeron la coloración para acetilcolinesterasa en el diagnóstico histológico de la EH, la que revela un marcado incremento de la actividad de la acetilcolinesterasa en un gran número de fibras nerviosas colinérgicas y tortuosos engrosamientos de las ramas nerviosas; pero, no es posible observar las neuronas.

Algunos investigadores piensan que el diagnóstico es confiable si se utiliza un examen morfológico de biopsias de mucosa teñidas con hematoxilina-eosina. Sin embargo, esto es difícil ya que los ganglios submucosos son muy pequeños y contienen de 3 a 5 células por ganglio, y se diseminan dentro del plexo de Meissner; más aún, existen diversos tipos de patologías como la displasia neuronal intestinal, la hipoganglionosis, la hiperganglionosis, la inmadurez de las células ganglionares y las desmosis colónica, que no pueden ser detectadas con la hematoxilina-eosina. ${ }^{5}$ Por esta razón, se debe utilizar coloraciones complementarias tales como la proteína S100, enolasa neuronal específica, catepsina, sinaptofisina, calretinina, cromogranina, proteína asociada a micro túbulos (MAP), para optimiza el diagnóstico. ${ }^{4,5,6}$

\section{RESULTADOS DEL TRATAMIENTO QUIRÚRGICO}

El tratamiento de esta enfermedad es quirúrgico, y existen diversas técnicas cuyo objetivo consiste en la resección del segmento agangliónico, y el descenso del segmento gangliónico el que se anastomosa con el conducto anal. ${ }^{7}$ $\mathrm{El}$ procedimiento quirúrgico de la $\mathrm{EH}$ ha evolucionado dramáticamente desde la disección recto sigmoidea (Swenson-Bill), a un "pouch" retrorrectal (Duhamel), una resección baja (Rehbein an Von Zimmerman), una disección endorrectal (Soave) y, recientemente, a una disección transanal y el descenso usando técnicas laparoscópicas. ${ }^{7,8}$

A pesar de la comprensión de la enfermedad por los avances en anatomía, fisiología y patología, los resultados de la cirugía no son perfectos. En Estados Unidos de Norteamérica se reporta que de 1000 casos nuevos de EH, más de la mitad de niños tratados con cirugía sufren de problemas crónicos como constipación, incontinencia, enterocolitis y/o dolor abdominal. ${ }^{9,10}$ Incluso en adultos, más de la mitad experimentará episodios ocasionales de incontinencia, y el $10 \%$ tendrá constipación severa que no responde al tratamiento médico. ${ }^{10}$

El Sawat et $\mathrm{al}^{11}$ reportan complicaciones postoperatorias de la $\mathrm{EH}$ en las que incluyen distensión abdominal y estreñimiento recurrente. El estreñimiento es probablemente la queja más común. Normalmente, los síntomas se desarrollan después de algunas semanas o meses de la operación. En la última década, varios investigadores han reconocido que los síntomas obstructivos son un problema importante, que ocurren en el 11-42\% de los niños después de lo que parece ser una operación técnicamente excelente. ${ }^{11}$

En un seguimiento de 21 años realizado por Wildhaber et $\mathrm{al}^{12}$, de 348 pacientes operados por EH, a 32 de ellos tuvieron que realizarles tardíamente miotomía o miectomía debido a estreñimiento. Concluyeron que la esfinterotomía fue moderadamente efectiva.

Engun y Grosfiel ${ }^{13}$, en el 2004, publicaron que muchos niños con $\mathrm{EH}$ tienen buen pronóstico inmediatamente después del tratamiento quirúrgico, pero en el seguimiento a largo plazo identificaron una serie de problemas como constipación, incontinencia y enterocolitis que perturba la calidad de vida.

Zimmer y Puri ${ }^{14}$, publicaron en el 2016, un metaanálisis, reportando que cerca del $15 \%$, de 800 pacientes, en quienes se les realizó pull-through endorectal transanal por 
$\mathrm{EH}$, continuaron presentando incontinencia y estreñimiento como problema principal. Sus conclusiones fueron: "estamos conscientes que nuestros datos del pronóstico a largo tiempo, como incontinencia, soiling (ensuciar la trusa con heces) y enterocolitis pueden ser subestimados de su incidencia real, comparados con otros reportes. Esta discrepancia sólo demuestra la falta de una evaluación segura a largo tiempo; por lo que recomendamos evaluar las funciones sexual y vesical en estos pacientes durante la niñez y la adultez".

Con respecto a las técnicas quirúrgicas y sus resultados a largo plazo, Bax ${ }^{15}$, en su artículo titulado "Incurabilidad de la enfermedad de Hirschsprung", manifiesta que los resultados a largo plazo con la técnica de Duhamel no son buenos cuando uno los mira críticamente, y que con las modernas técnicas de abordaje transanal con asistencia laparoscópica los resultados no parecen diferir mucho con aquellos del procedimiento clásico. Refiere algo importante respecto a la evaluación del esfínter interno con la manometría: existe una gran diferencia del esfínter interno en el paciente con EH y el normal. En la EH el reflejo inhibitorio recto anal está ausente, y este reflejo permanece ausente aún después de la cirugía. Bax concluye diciendo: "la cirugía no cura completamente la EH, asi que se debe dejar de hablar de resultados perfectos". ${ }^{15}$

Otro estudio comparativo entre las técnicas laparoscópicas transabdominal y transrectal para el descenso abdominoperineal en $\mathrm{EH}$, reportó que, de 52 niños mayores de tres años, 28 paciente fueron operados mediante TEPT (pull-through endorectal transanal), y 24 con LEPT (pull-through endoscópico asistido con laparoscopía). Los pacientes fueron seguidos a lo largo de 5,7 años para TEPT y de 10,1 años para LEPT. Veintinueve pacientes presentaron soiling al final del seguimiento. No hubo diferencias significativas entre ambas técnicas (54\% y 58 $\%)^{3}$. Tampoco lo hubo en cuanto al estreñimiento 11 casos $(24 \%$ y $17 \%) .{ }^{16}$.

Finalmente, Levitt y Peña ${ }^{3}$, en el 2012, publicaron un artículo sobre complicaciones posoperatorias en la EH, en el que concluyeron: "Nosotros no comprendemos totalmente la fisiopatología de la EH, especialmente la hipomotilidad, no obstante, la presencia de las células ganglionares en el colon descendido".

\section{LA ENFERMEDAD DE HIRSCHPRUN TODAVÍA ES DESCONOCIDA}

La EH es una entidad que todavía no se ha logrado comprender en su integridad. En los últimos años se está descubriendo la importancia del estudio genético y se está avanzando en la comprensión del rol de estructuras histológicas como las células intersticiales de Cajal, de las células productoras de sustancias paracrinas o neurócrinas de tipo no adrenérgico y no colinérgico que intervienen en la relajación del intestino.

Además del sistema nervioso entérico, las células intersticiales de Cajal (ICC) tienen un rol importante en la generación del peristaltismo gastrointestinal, rol que en nuestro medio no se le ha dado la debida atención. Se conoce que las células intersticiales de Cajal cumplen las siguientes funciones en el intestino: La más importante es la generación de ondas lentas que permiten a estas células actuar como células pacemaker en el interior de las capas musculares del intestino; además, actúan como reguladoras de la actividad mecánica y de la neurotransmisión controlando el cronotropismo y el inotropismo en el músculo liso. Estudios sobre las ICC en la $\mathrm{EH}$ han demostrado que en el intestino agangliónico, el total de preparaciones y secciones congeladas mostraron ICC escasas o únicas a nivel del plexo mientérico entre las capas circular y longitudinal. Estas células son delgadas y bipolares, y están estrechamente relacionadas con los plexos nerviosos hipertróficos. ${ }^{17,18}$.

\section{Neurotransmisores y neuromoduladores}

El avance en el estudio de los neurotransmisores a nivel del tracto intestinal ha llevado a sugerir la hipótesis de que la contracción del colon en la EH podría ser el resultado de una aplasia de los nervios simpáticos, los cuales inhiben normalmente las contracciones del intestino. ${ }^{19}$

Se conocen las acciones de ciertos neurotransmisores denominados no adrenérgicos no colinérgicos como el VIP (péptido vaso intestinal) y el NO (óxido nítrico), los cuales relajan el esófago terminal y la porción proximal receptiva del estómago, que son producidos por la inervación vagal. ${ }^{20}$ Por otro lado, se ha estudiado el efecto relajante de ciertas sustancias como el ATP (adenosín trifosfato) y sus derivados adenosina y otros ubicados sobre las fibras musculares lisas. El ATP (adenosín trifosfato) es el mayor neurotransmisor purinérgico liberado por las neuronas inhibitorias que relajan el músculo liso. Se cree que el ATP es el responsable de la hiperpolarización la que se produce rápidamente y que está correlaciona con una relajación mecánica fásica no sostenible. ${ }^{21}$ Existen dos tipos de receptores purinérgicos: los P1 (para la adenosina) y los P2 para el ATP. Dentro de los receptores P2 para el ATP se han encontrado subtipos como los P2Y, los cuales son los mediadores de la relajación en diversas partes del tracto digestivo $^{21}$

También existen péptidos como el CGRP (péptido relacionado con la liberación de gastrina), somatostatinas, encefalinas, bombesina, galanina, prostanoides y serotonina, cuyos roles no ha sido ampliamente estudiado en la EH. Se sabe que existe disminución de la sustancia $\mathrm{P}$ en el segmento agangliónico. También se ha encontrado aumento del NPY (neuropéptido Y) en el segmento agangliónico, lo que significaría incremento de las fibras nerviosas extrínsecas, que se sabe están hipertrofiadas en dicho segmento y por lo tanto puede servir de ayuda en el diagnóstico de la aganglionosis del colon. ${ }^{22}$

\section{CAUSAS GENÉTICAS DE LA ENFERMEDAD DE HIRSCHPRUNG}

Actualmente los investigadores admiten la hipótesis genética como una de las causas de la EH. Los argumentos a 
su favor son: la existencia de familias con esta enfermedad, el aumento de la incidencia en ciertas poblaciones, la asociación con otras anomalías cromosómicas (trisomía 21) y por la mayor incidencia en el sexo masculino. ${ }^{17}$

Se han encontrado muchos genes implicados en la EH entre los más importantes podemos mencionar: El protooncógen RET. Es un gen autosómico dominante que se encuentra en el cromosoma 10. La mutación de este gen ha sido estudiada en el ratón transgénico RET-K. En el ser humano, la mutación del RET se ha encontrado en el 10 al $15 \%$ de las formas recto sigmoideas y en e $50 \%$ de las formas extendidas de la $\mathrm{EH}$.

El gen RET es un receptor que regula la actividad de factores de crecimiento sobre las neuronas intestinales. Por lo tanto, es el primer gen que debería incluirse en el estudio genético tras diagnosticarse la EH. Lorente y $\mathrm{col}^{23}$ reportan que de 21 casos de EH, en 17 las mutaciones en el gen $R E T$ fue el hallazgo más frecuente, asociado a otras dos nuevas modificaciones relacionadas al gen $\mathrm{PHOX} 2 \mathrm{~B}^{23}$.

La importancia del estudio genético en la evaluación de la EH radica en las implicaciones terapéuticas y pronosticas, no sólo en caso de enfermedad aislada sino también en enfermedades asociadas.

La importancia terapéutica y pronostica radica en que las mutaciones en ciertas regiones del gen RET se asocian al síndrome MEN 2A o MEN 2B, asociados a un riesgo mayor de carcinoma medular de tiroides, hiperplasia de las glándulas paratiroides y feocromocitoma. El MEN 2B se caracteriza por presentar, en la primera infancia, carcinoma medular de la tiroides (CMT) y feocromocitoma (frecuencia del $50 \%$ ). Casi nunca se observa hiperparatiroidismo en las personas con MEN 2B. Los pacientes con MEN 2B presentan con frecuencia otras características físicas, como ser altos y delgados, tener pequeños tumores benignos (no cancerosos) en los labios y la lengua y una enfermedad en la que se engruesa e irrita el intestino grueso. ${ }^{2}$

\section{ESTRATEGIA FRENTE A UN PACIENTE CON ENFERMEDAD DE HIRSCHPRUNG}

Si la EH, como hemos podido darnos cuenta, es una entidad que aún no se ha logrado determinar todos los factores que intervienen en su patogénesis; entonces, ¿Qué debemos hacer frente a un recién nacido o preescolar en el que se sospecha EH?

Consideramos que es necesario aplicar el protocolo planteado por Martuciello ${ }^{5}$, con la finalidad de tener mayor certeza en la aproximación al diagnóstico, sin que esto quiera decir que se logre el $100 \%$ de seguridad en el mismo. En el neonato, el protocolo se inicia realizando una biopsia rectal complementando con coloraciones histoquímicas (ACHE, NADPH, LDH, SDH). En la cual se debe tener en consideración lo siguiente:

1. Si se encuentra un patrón ACHE tipo A con fibras nerviosas prominentes en toda la lámina propia, indicaría el hallazgo clásico de la EH.

2. Si se encuentra el patrón tipo $B$, fibras similares al patrón A se observan solo en la muscularis mucosae, adyacente a la lámina propia. Esto es característico en el periodo neonatal; pero en dos semanas el paciente sospechoso puede cambiar al patrón A. De esto resultará una EH positiva o negativa. Si resulta positiva, se realizará un enema contrastado para mayor información con fines quirúrgicos.

3. Se deberán hacer estudios moleculares para determinar mutaciones del gen RET, que juega un rol en dos criptopatías el MEN 2A (Neoplasia endocrina múltiple tipo 2A) y el MEN 2B, y también en el carcinoma tiroideo medular familiar (FMTC).

4. A todos los pacientes con EH se les debe hacer un seguimiento y vigilancia con pruebas bioquímicas para mutaciones MEN y MTC, y en otros miembros susceptibles de la familia, ya que ellos están en riesgo de desarrollar tumores neuroendocrinos.

4. Si el resultado fuese negativo para $\mathrm{EH}$, se indica manometría y se realiza una biopsia de grosor total del intestino, a través de laparoscopía transumbilical solo con un trocar. ${ }^{5}$

5. Se realizará estudios histoquímicos para ACHE, LDH, ANE SDH durante el preoperatorio.

6. Durante el transoperatorio se investigará la presencia de enzimas como ANE (alpha-naphthylesterase), y $\mathrm{NADPH}$, que permiten una evaluación clara y rápida de la aganglionosis y del segmento hipogangliónico que requieren resección.

Con la introducción de la biopsia intestinal de grosor total por vía laparoscópica, este protocolo cubre el criterio de otros diagnósticos dentro de la presentación de un seudo Hirschsprung como el hipo- aganglionosis y la displasia neuronal intestinal. ${ }^{5}$

7. En caso de hipoganglionosis se realizará la extirpación del segmento colónico afectado (colectomía subtotal) de acuerdo a la extensión del segmento afectado ${ }^{25}$

8. En caso de displasia intestinal tipo $\mathrm{B}$, el tratamiento está influenciado por los diversos tipos de manifestaciones clínicas. En los casos de constipación leve, sin complicaciones sistémicas o síntomas obstructivos, se tiende a tratar con medidas conservadoras. La mayoría de los casos pueden resolverse espontáneamente a la edad de cuatro años, debido a la maduración del sistema nervioso entérico.

Por otro lado, la displasia neuronal intestinal tipo B, con constipación severa, síntomas obstructivos e infecciosos, requerirá un tratamiento más invasivo. El tratamiento quirúrgico consiste en la resección intestinal, y deberá estar reservado para los casos refractarios al manejo clínico por lo menos de seis meses de duración. ${ }^{26}$

Queremos hacer referencia a una entidad clínica conocida con el nombre de desmosis colónica que complica más el diagnóstico y tratamiento de la EH. La desmosis colónica, en contraste con la EH, la hipoganglionosis, la displasia neuronal, y la inmadurez del plexo, es una enfermedad constipante del colon con el sistema nervioso vengativo intacto, en la que se observa ausencia de la red o malla del tejido conectivo que enlaza las fibras musculares circulares 
y longitudinales del intestino, principalmente en la muscularis propia, y que conduce a hipoperistalsis. Debido a la hipoperistalsis, que se produce como consecuencia de la falla en la coordinación de estos movimientos por falta del tejido conectivo, cuya función es limitar la dilatación del colon, en la adultez produce un megacolon gigante.

Finamente, debemos referir que Maier-Ruge ${ }^{27,28}$, confirmó la existencia de la desmosis colónica al estudiar 206 casos de niños con aparente normalidad en la inervación intestinal que padecían de estreñimiento e hizo coloraciones de las biopsias con hematoxilina eosina y rojo de ácido pícrico/ sirius, encontrando 14 casos en los que les faltaba la red de tejido conectivo entre las capas musculares circular y longitudinal.

\section{CONCLUSIÓN}

Por lo expuesto, podemos concluir que la $\mathrm{EH}$ es una entidad aún desconocida por carecer de patrones histológicos definitivos. Para el diagnóstico solo se toma en cuenta la ausencia de los plexos nerviosos de Auerbach y Meissner; sin embargo, no se conoce en su totalidad las funciones de las células de Cajal, y de diversas sustancias adrenérgicas, colinérgicas, y purinérgicas que intervienen en el mecanismo de contracción y relajación intestinal.

Estas deficiencias en el conocimiento de la EH explicarían los resultados no del todo satisfactorios de la cirugía, tanto a nivel nacional como internacional, lo que constituye un reto para todos los cirujanos pediatras, sobre cuyos hombros recae la responsabilidad del diagnóstico y tratamiento de los afectados por este problema, y para que, con la intervención, alcancen una mejor calidad de vida.

\section{REFERENCIAS BIBLIOGRÁFICAS}

1. Knott D, Agrawel R. Hirschsprung disease. URL disponible en: https://radiopaedia.org/articles/hirschsprung-disease

2. De Manueles Jiménez J. Enfermedad de Hirschsprung. URL disponible en: https://www.aeped.es/sites/default/files/ documentos/6-hirschsprung.pdf

3. Levitt MA, Dickie B, Peña A. The Hirschsprung's patient who is soiling after what was considered a "successful" pull-through. Seminars in Pediatric Surgery. (2012) 21, 344-353a

4. Ridaura-Sanz, Carrasco-Daza D. Biopsia del tubo digestivo bajo. Acta Pediatr Mex. 2010;31(2):78-86.

5. Martucciello G. Hirschsprung's disease, one of the most difficult diagnosis. Eur J Pediatr Surg. 2008; 18: 140-149.

6. Carrasco-Daza D. Biopsia del tubo digestivo bajo. Acta Pediatr Mex. 2010;31(2):78-86

7. De la Torre L, Ortega J. Pull through endorectal transanal para la enfermedad de Hirschsprung. J Pediatr Surg. 1998;33: 1283-86

8. Peña A, Levitt M. The Surgical treatment of Hirschsprung disease. Constipation. 2009 :221-234.

9. Calkins MC. Hirschsprung disease beyond infancy. Clin Colon Rectal Surg. 2018;31:51-60.

10. Rintala RJ1, Pakarinen MP. Long-term outcomes of Hirschsprung's disease. Long-term outcomes of Hirschsprung's disease. Semin Pediatr Surg. 2012 Nov;21(4):336-43.
11. El-sawaf M.I., Coran A.G., Teitelbaum D.H. (2008) Reoperative Surgery for Hirschsprung Disease. In: Teich S, Caniano DA. (eds) Reoperative Pediatric Surgery. Humana Press. https://doi. org/10.1007/978-1-60327-071-7_17

12. Wildhaber BE, Pakarinen M, Rintala RJ, Coran AG, Teitelbaum DH. Posterior myotomy/myectomy for persistent stooling problems in Hirschsprung's disease. J Pediatr Surg. 2004; 39: 920-926

13. Engun A. S. Grosfield J L. Long terms results of treatment of Hirschsprung disease. Seminars in Pediatric Surgery. 2004;13 (4):273-85.

14. Zimmer J, Tomuschat C, Puri P. Long-term results of transanal pullthrough for Hirschsprung's disease: a meta-analysis. Pediatr Surg Int. 2016;32(8):743-9.

15. Bax K(N)MA. Duhamel Lecture: The incurability. Eur J Pediatr Surg 2006; $16: 380-384$.

16. Stensrud JE, Emblem R, Biornland K. Functional outcome after operation for Hirschsprung disease transanal vs transabdomial approach. Journal of Pediatric Surgery. 2010;45,1640-44.

17. Rolle U; Piasecina Piotrowska A, Nemeth L; Puri P. Altered distribution of interstitial cells of Cajal in Hirschsprung disease. Arch Pathol Lab Med. 2002, (12):928-933.

18. Romero- Trujillo J.O. Sistema nervioso entérico y motilidad gastrointestinal. Acta Pediatr Mex. 2012;33(4):207-214.

19. Gannon, JB , H R Noblett, Burnstock G Adrenergic innervation of bowel in Hirschsprung disease. Br Med J. 1969; 2:330-331.

20. Lefebrvet RA. Non cholinergic non adrenergic neurotransmitter in the proximal stomach-AD https://www.sciencedirect.com/sdfe/ pdf/download/eid/1-s2.0-030636239390301D/first-page-pdf

21. Gallego D, Muñe N, Gil V, Martínez Cutilla M, Jiménez M. Mecanismos responsables de la relajación neuromuscular en el tracto gastrointestinal. Rev Esp Enf Dig. ,2016;108 (11):721-723.

22. Gutierrez 0. Péptidos reguladores del sistema endocrino. Acta Med Colomb. 1988; 13:254 -258.

23. Lorente M, Miren RA, Sánchez Galán A. Aminoso C, García Pablo S, Lapunzina, Solera - García J. Nuevas mutaciones asociadas a la enfermedad de Hirschsprung. Anales de Pediatría. URL disponible en: https://www.sciencedirect.com/science/article/pii/ S1695403319304242

24. Medical Center: Neoplasias endocrinas múltiples. URL disponible en: https://mdanderson.es/elcancer/elcancerdelaaalaz/neoplasiasendocrinas-multiples

25. Hong Yi, Zhang Hong, Jie-Xiong Feng, Lei Huang, et al. Diagnosis and surgical treatment of isolated hypoganglionosis. World $J$ Pediatr. 2008 Nov;4(4):295-300.

26. Toledo de Arrua P.L, Antunes-Terra S, Paiva-Ortolan EV, Marchesan Rodrigues MA. Intestinal neuronal dysplasia type B: A still little known diagnosis for organic causes of intestinal chronic constipation. World J Gastrointest Pharmacol Ther. 2016.

27. Meier-Ruge W. A desmosis of the colon: A working hypothesis of primary chronic constipation. Eur J Pediatr Surg. 8 (1998) 299-303.

28. Szymańska S, Markiewicz M-Kijewska, Pyzlak, M, A Wieckowska Karkucinska, A, Kalicinski P, Grajkowska W, Pronicki M Pathogenesis of chronic constipation in a Polish group of paediatric patients -an attempt to create the optimal histopathological diagnostic protocol. Prz Gastroenterol. 2019 May;22(3):214-7.

Conflicto de interés: Ninguno según los autores. Financiamiento: Por los autores.

\section{CORRESPONDENCIA}

Emiliano Paico Vílchez

epaicov@gmail.com

Fecha de recepción: 16-09-2020.

Fecha de aceptación: I6-II-2020. 